\title{
Oscillating Purkinje Neuron Activity Causing Involuntary Eye Movement in a Mutant Mouse Deficient in the Glutamate Receptor $\delta 2$ Subunit
}

\author{
Takashi Yoshida, ${ }^{1,2 \star}$ Akira Katoh, ${ }^{1,2 \star}$ Gen Ohtsuki, ${ }^{1,2}$ Masayoshi Mishina, ${ }^{2,3}$ and Tomoo Hirano ${ }^{1,2}$ \\ ${ }^{1}$ Department of Biophysics, Graduate School of Science, Kyoto University, Kyoto 606-8502, Japan, ${ }^{2}$ Core Research for Evolutional Science and Technology, \\ Japan Science and Technology Agency, Saitama 332-0012, Japan, and 3Department of Molecular Neurobiology and Pharmacology, School of Medicine, \\ University of Tokyo, and Solution Oriented Research for Science and Technology, Tokyo 113-0033, Japan
}

\begin{abstract}
How failures in regulation of synaptic transmission in the mammalian CNS affect neuronal activity and disturb motor coordination is addressed. The mutant mouse deficient in the glutamate receptor $\delta 2$ subunit, specifically expressed in cerebellar Purkinje neurons, has defects in synaptic regulations such as synaptic plasticity, stabilization, and elimination of synaptic connections and shows failures in motor coordination and learning. In this study, the cause of motor discoordination of the $\delta 2$ mutant mouse was analyzed by comparing its motor control ability with those of the wild-type mouse and the lurcher mutant mouse, which loses all Purkinje neurons, the sole output neurons in the cerebellar cortex. Unexpectedly, the $\delta 2$ mutant mouse showed severer motor discoordination than the lurcher mouse without any cerebellar cortical outputs. The $\delta 2$ mutant mouse showed involuntary spontaneous eye movement with characteristic $10 \mathrm{~Hz}$ oscillation, which disappeared by ablation of the cerebellar flocculus, suggesting that the $\delta 2$ mutant cerebellar cortex outputs an abnormal signal. In vivo extracellular recordings of neuronal activity revealed that Purkinje neurons tended to fire clustered action potentials and complex spikes at $\sim 10 \mathrm{~Hz}$ in the $\delta 2$ mutant mouse. A whole-cell patch-clamp recording from Purkinje neurons in cerebellar slices indicated that the clustered action potentials could be induced by climbing fiber activation. Taken together, our results suggest that the $\delta 2$ subunit deficiency produces the oscillating activity in Purkinje neurons by enhancing climbing fiber inputs, causing surplus movement and affecting motor control worse than no signal at all.
\end{abstract}

Key words: Purkinje cell; motor control; glutamate receptor; knock-out mouse; oscillation; eye movement; $\delta 2$ subunit; climbing fiber

\section{Introduction}

The cerebellum is implicated in motor control and learning. Previous studies on mutant mice revealed that deficits in synaptic regulations such as synaptic plasticity, synapse formation, and elimination in the cerebellar cortex are accompanied with motor discoordination and motor learning failures (Aiba et al., 1994; Conquet et al., 1994; Chen et al., 1995; Kashiwabuchi et al., 1995; Shibuki et al., 1996; De Zeeuw et al., 1998; Katoh et al., 2000). However, how synaptic regulation failures cause behavioral abnormality has been elusive. We addressed this issue working on two strains of mice with mutations on an orphan receptor protein related to the ionotropic glutamate receptors (GluRs), which are specifically expressed in Purkinje neurons (PNs), the sole output neurons in the cerebellar cortex.

GluRs are classified into AMPA, kainate, and NMDA subtypes

Received March 17, 2003; revised Dec. 11, 2003; accepted Jan. 13, 2004.

This work was supported by grants from the Ministry of Education, Culture, Sports, Science and Technology, Japan to T.H. We thank Drs. S. Nakanishi, Y. Sakurai, K. Funabiki, Y. Hirata, and M. Kengaku for comments on this manuscript.

*T.Y. and A.K. contributed equally to this work.

Correspondence should be addressed to Dr. Tomoo Hirano, Department of Biophysics, Graduate School of Science, Kyoto University, Sakyo-ku, Kyoto 606-8502, Japan. E-mail: thirano@nb.biophys.kyoto-u.ac.jp.

DOI:10.1523/JNEUROSCI.0783-03.2004

Copyright $\odot 2004$ Society for Neuroscience $\quad$ 0270-6474/04/242440-09\$15.00/0
(Hollmann and Heinemann, 1994). These receptors are heteromeric tetramers composed of subunits that have been identified by molecular cloning studies (Hollmann and Heinemann, 1994; Rosenmund et al., 1998). The $\delta 2$ subunit (GluR $\delta 2$ ) has been classified into a novel subgroup of GluR subunits based on amino acid sequence (Araki et al., 1993; Lomeli et al., 1993). GluR $\delta 2$ is selectively localized at postsynaptic membrane apposed to glutamatergic parallel fiber terminals (Takayama et al., 1996; Zhao et al., 1997) and is required for the induction of long-term depression (LTD) (Hirano et al., 1994, 1995; Kashiwabuchi et al., 1995; Jeromin et al., 1996), a form of synaptic plasticity implicated in motor learning (Linden and Connor, 1995; Raymond et al., 1996; Ito, 2001). The mutant mouse deficient in GluR $\delta 2$ (DM) lacks LTD and shows motor discoordination and motor learning deficits (Funabiki et al., 1995; Kashiwabuchi et al., 1995; Kishimoto et al., 2001). In a DM, the number of synapses between parallel fibers and a PN is reduced, and a PN is innervated by multiple climbing fibers (Kurihara et al., 1997; Hashimoto et al., 2001; Ichikawa et al., 2002). In an adult wild-type mouse (WM), a PN is innervated by a single climbing fiber (Ito, 1984). However, how GluR $\delta 2$ is involved in LTD induction, how the GluR $\delta 2$ deficiency reduces the number of parallel fiber-PN synapses, and how it causes motor discoordination have not been elucidated. On the other hand, the lurcher mutant mouse (LM) has a genetic muta- 
tion resulting in a single amino acid replacement in the GluR $\delta 2$ gene (Zuo et al., 1997). The mutated GluR $\delta 2$ forms ion channels that allow cations to permeate in the basal condition and kills all PNs during postnatal development (Swisher and Wilson, 1977; Zuo et al., 1997; Kohda et al., 2000). An LM also shows motor discoordination and motor learning deficits (Fortier et al., 1987; Hilber and Caston, 2001). Thus, DMs and LMs provide a unique opportunity to directly compare behavioral effects of synaptic regulation failures with those of complete ablation of cerebellar cortical outputs, both of which are induced by mutations on the same protein. Here, we have examined motor control ability of DMs and LMs and analyzed the cause of severe motor discoordination in DMs.

\section{Materials and Methods}

Animals. The strain of WMs was C57BL/6. DMs were generated as described previously (Kashiwabuchi et al., 1995), and LMs (B6CBACaA ${ }^{\mathrm{W}-\mathrm{J} /}$ AGrid2 ${ }^{\mathrm{LC}}$ ) were obtained from The Jackson Laboratory (Bar Harbor, ME). DMs and LMs were backcrossed to the C57BL/6 strain for 13 and at least 3 generations, respectively. Thus, DMs and LMs had a predominantly C57BL/6 genetic background (DM, >99.99\%; LM, >93\%). Twoto 3-month-old mice were used for experiments, unless stated otherwise. The experimental procedure was performed in accordance with the guidelines regarding the care and use of animals for experimental procedures of the National Institutes of Health and Kyoto University and was approved by the local committee for handling experimental animals in the Graduate School of Science at Kyoto University.

Eye movement recording. The method of eye movement recording was similar to that described previously (Iwashita et al., 2001). Here, the sampling frequency of eye position recording was $60 \mathrm{~Hz}$, and the estimation of eye movement amplitude was recalibrated in accordance with a previous study (Stahl et al., 2000).

Single-unit recording. Under ketamine and xylazine anesthesia, a head holder was fixed to the skull of a mouse by embedding three small screws in dental cement. A small hole $(<1.0 \mathrm{~mm})$ was drilled in the occipital bone above the flocculus ( $4.5 \mathrm{~mm}$ caudal, $3.0 \mathrm{~mm}$ lateral from bregma), and the dura was exposed and covered with Vaseline and a piece of polyvinyl chloride film. After the surgery, the mouse was allowed to recover for $>48 \mathrm{hr}$. To record the $\mathrm{PN}$ activity, the mouse was fixed on the recording chamber using the head holder under light anesthesia with diethyl ether, and the dura was partly removed with great care. We then waited until the mouse was aroused. The arousal was confirmed by excretion and limb movement. Extracellular recordings of the PN activity was performed with a glass micropipette filled with $2 \mathrm{M} \mathrm{NaCl}(3-5 \mathrm{M} \Omega$ ). The electrode tip was positioned above the small hole and moved downward using a micromanipulator. The signal was amplified, low-pass filtered at $10 \mathrm{kHz}$ and high-pass filtered at $100 \mathrm{~Hz}$, recorded using a digital tape recorder, and analyzed offline with the Labview system (National Instruments, Austin, TX) later. The sampling rate for analysis was 20 $\mathrm{kHz}$. Simple spikes were identified by the amplitude, and complex spikes were identified visually by the characteristic waveform. Action potentials generated by excitatory inputs at synapses formed between a climbing fiber and distal dendrites of a PN in a DM might have been identified as simple spikes (Hashimoto et al., 2001). The recording site was identified as follows. Dextran-Texas Red (1\%; Molecular Probes, Eugene, OR) dissolved in $2 \mathrm{M} \mathrm{NaCl}$ was applied to the cerebellum from the glass micropipette after recording. Three millisecond voltage pulses of $80 \mathrm{~V}$ (both positive and negative) were delivered at $100 \mathrm{~Hz}$ for $\sim 10 \mathrm{~min}$. Then, the mouse was anesthetized deeply and perfused with $0.1 \mathrm{M}$ phosphate buffer, $\mathrm{pH} 7.4$, containing $4 \%$ paraformaldehyde. The $50 \mu \mathrm{m}$ slices of cerebellum were prepared by a cryostat after being embedded in optimal cutting temperature compound (Tissue-Tek, Torrance, CA) and observed with a fluorescent microscope.

Simultaneous recording of single-unit activity and eye movement. Simultaneous recording of the eye position and the electrophysiological singleunit activity was performed using a common external trigger. The low sampling frequency $(60 \mathrm{~Hz})$ of our eye position recording system ham-
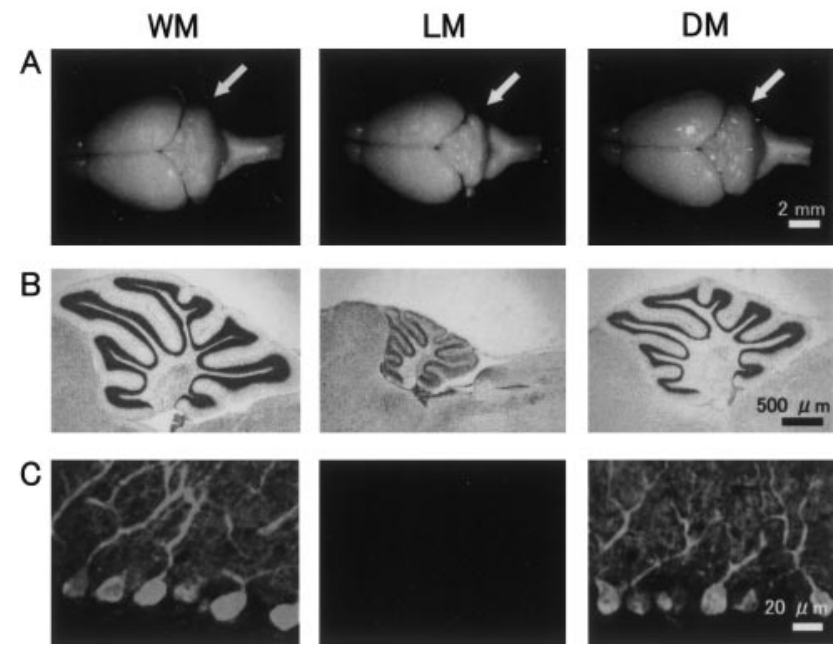

Figure 1. The cerebellar morphology of WMs, DMs, and LMs. $A-C$, Dorsal views of whole brains $(A)$ and saggital slices of cerebella $(B, C)$. Each arrow indicates the cerebellum. The cerebellum of an LM was the smallest, and that of a DM was slightly smaller than that of a WM. The cerebellar slices were stained with cresyl violet $(B)$ or with anti-calbindin antibody $(C)$. Calbindin was specifically expressed in PNs within the cerebellar cortex.

pered the precise timing adjustment. The eye position recording was delayed for 4-25 msec. After the simultaneous recording, the location of the recording site within the flocculus was determined by electrical stimulation ( $3 \mathrm{msec}, 80 \mathrm{~V}$ pulses at $100 \mathrm{~Hz}$ for $1 \mathrm{sec}$ ) through the recording electrode, which induced horizontal, vertical, or no eye movement. The single-unit activity was analyzed in relation to the induced eye movement (either horizontal or vertical).

Patch-clamp recording. The slice patch-clamp recording was performed essentially as described previously (Kashiwabuchi et al., 1995). Horizontal slices $(200 \mu \mathrm{m})$ cut from the cerebellum of mice at postnatal days $22-28$ were perfused continuously at $22-24^{\circ} \mathrm{C}$ with an external solution consisting of $124 \mathrm{~mm} \mathrm{NaCl}, 26 \mathrm{mM} \mathrm{NaHCO}_{3}, 1.8 \mathrm{~mm} \mathrm{KCl,} 1.24 \mathrm{~mm}$ $\mathrm{KH}_{2} \mathrm{PO}_{4}, 2.5 \mathrm{~mm} \mathrm{CaCl}_{2}, 1.3 \mathrm{~mm} \mathrm{MgCl}$ and $10 \mathrm{~mm}$ glucose and saturated with $95 \% \mathrm{O}_{2}$ and $5 \% \mathrm{CO}_{2}$ in the presence of $20 \mu \mathrm{M}$ bicuculline. A glass micropipette filled with an internal solution consisting of $140 \mathrm{~mm} \mathrm{KCl}, 5$ mM EGTA, and 10 mм HEPES, pH7.3, was used for patch-clamp recordings. The electrode resistance was 3-4 M $\mathrm{M}$. Cell-attached, whole-cell voltage-clamp and current-clamp recordings were performed with a patch-clamp amplifier (EPC-9; Heka, Lambrecht, Germany). The junction potential was offset, and series resistance compensation was optimized. A glass pipette filled with the external solution was placed on a granular layer and used to stimulate a climbing fiber. The in vitro patchclamp recording was performed in a slice prepared from the cerebellar hemisphere, because we recorded the clustered firing and the $10 \mathrm{~Hz}$ oscillation not only from the flocculus but also from the hemisphere in vivo. The lower recording temperature in the slice experiment is likely to affect the firing frequency of a $\mathrm{PN}$, which was smaller than that in vivo.

Histology. Cerebellar slices (20 $\mu \mathrm{m}$ thick) were prepared as described above for cresyl violet staining and immunohistochemistry with an anticalbindin antibody (Swant, Bellinzona, Switzerland) and a secondary anti-IgG antibody conjugated with Alexa488 (Molecular Probes).

\section{Results}

\section{Morphology and motor coordination}

We first reexamined the cerebellar morphology of DMs, LMs, and WMs. In this study, we used mice backcrossed to the C57BL/6 strain to minimize the variation of genetic background. As reported previously, PNs were absent, and the cerebellum was small and severely deformed in an LM, as shown in Figure 1 (Swisher and Wilson, 1977). In contrast, the cerebellum of a DM looked almost normal and indistinguishable from that of a WM at a gross anatomical level, although it has been reported that the 
A

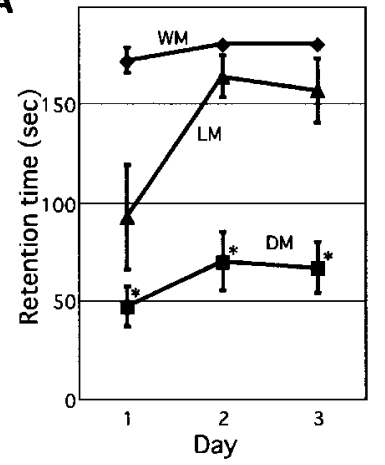

B

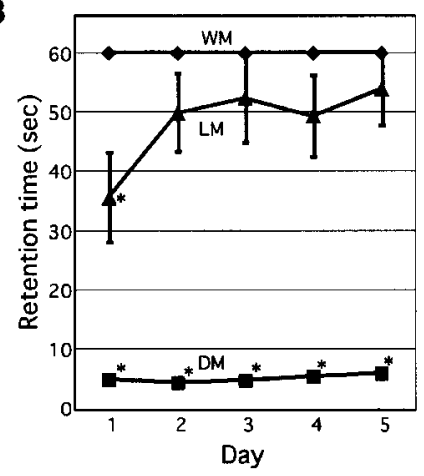

Figure 2. Motor control of WMs, DMs, and LMs. The retention times during which a mouse stayed on a stationary $\operatorname{rod}(A)$ or on a rotating $\operatorname{rod}(B)$ are shown (mean $\pm S E M)$. Each trial for a stationary rod test was $180 \mathrm{sec}$, and that for a rotating rod test was for $60 \mathrm{sec}$. The rotating rod test was started $1 \mathrm{~d}$ after the last day of the stationary rod test. Three rotating rod trials were performed each day, and the longest retention time was used to calculate the mean. $n=7$ $(\mathrm{WM}) ; n=11(\mathrm{DM}) ; n=7$ (LM). *Significantly lower than WMs ( $p<0.05$; Steel Dwass's test).

number of synapses between parallel fibers and a PN is reduced and a PN is innervated by multiple climbing fibers in a DM (Kashiwabuchi et al., 1995; Kurihara et al., 1997; Hashimoto et al., 2001; Ichikawa et al., 2002). PNs formed well-developed dendrites in a DM as those in a WM (Fig. 1C).

Next, motor coordination of these mutant mice was evaluated by measuring how long they could stay on a stationary or rotating rod (3 cm diameter) (Fig. 2). Unexpectedly, LMs showed much better performance than DMs. On the last day of the trial, WMs, LMs, and DMs stayed on a stationary rod for $180 \mathrm{sec}$ (the possible maximum value in the trial; $n=7$ ), $154 \pm 16 \mathrm{sec}$ (mean \pm SEM; $n=7)$, and $67 \pm 13 \mathrm{sec}(n=11)$ respectively, and on a rod rotating at $10 \mathrm{rpm}$ for $60 \mathrm{sec}$ (the possible maximum value), $54 \pm$ $16 \mathrm{sec}$, and $6 \pm 1 \mathrm{sec}$, respectively. The score of DMs was significantly lower than those of WMs and LMs $(p<0.05$; Steel Dwass's test). These results suggest that motor coordination is more severely impaired in DMs than in LMs.

\section{Spontaneous eye movement}

To further evaluate the motor control ability of DMs and LMs, we measured spontaneous eye movement (Stahl et al., 2000; Iwashita et al., 2001). The eye position of a mouse in the resting state was monitored by a video camera in light. In a WM and an LM, the eye rarely moved. In contrast, the eye moved around irregularly and continuously in a DM (Fig. 3A). Thus, a DM showed the surplus eye movement. To further analyze the spontaneous eye movement, the spectral analysis was performed. The power spectrum represents the contribution of each frequency component to the eye movement (Fig. 3B). The power value was high at a low frequency and tended to decline with the increase in frequency in any mice examined. The power spectra of spontaneous eye movement in WMs and LMs were similar. In contrast, the power value in DMs was higher than the others and had peaks at $\sim 1$ and $10 \mathrm{~Hz}$ (Fig. $3 B$, arrowhead and arrow), indicating that the spontaneous eye movement of DMs is larger than that of WMs or LMs at low and high frequencies. In the dark, a DM also showed substantial spontaneous eye movement (Fig. 3A), but both 1 and $10 \mathrm{~Hz}$ components were less clear in the power spectrum (Fig. 3B), suggesting that visual inputs contribute to the generation of the oscillation at these frequencies.

We thought that the surplus eye movement in a DM might be caused by the inappropriate neuronal signal generated in the cer-

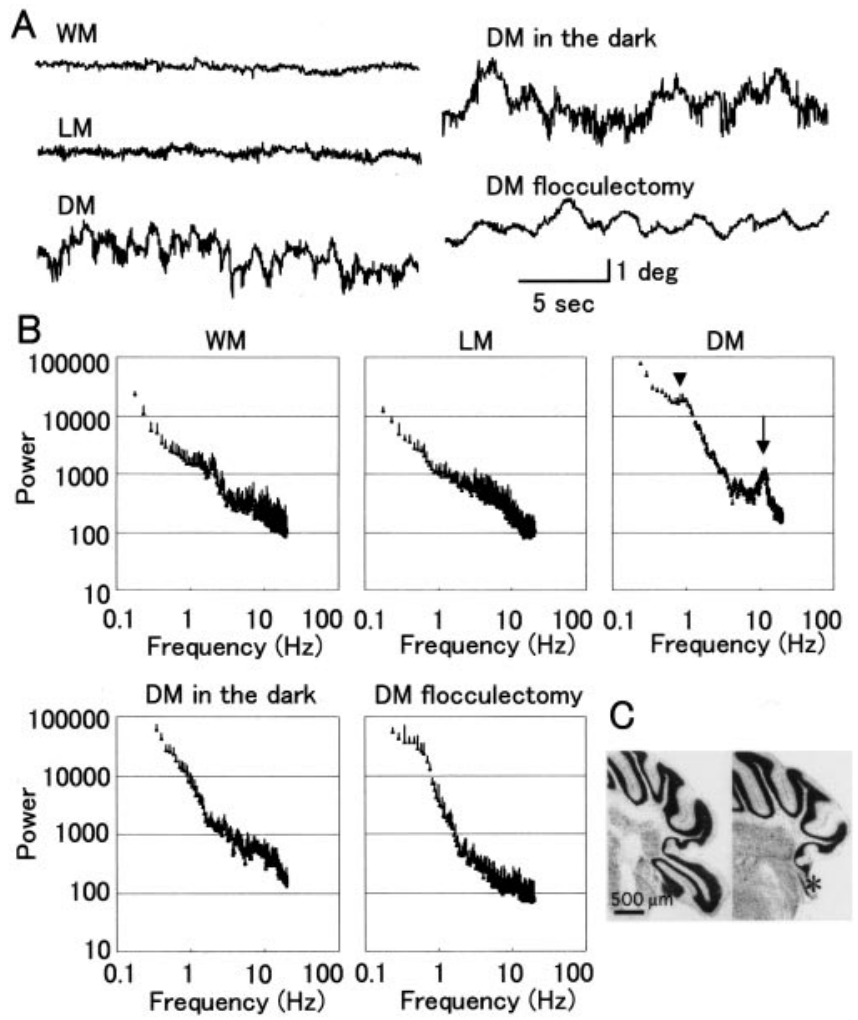

Figure 3. The spontaneous horizontal eye movement of mice. A, Traces show the horizontal eye position. $B$, The power specrtra calculated from eye position recordings. The spectra from 9-12 mice were averaged and then smoothed (Microsoft Excel). An arrowhead indicates the peak $\sim 1 \mathrm{~Hz}$, and an arrow indicates that at $\sim 10 \mathrm{~Hz}$. The mean \pm SEM is shown at each frequency. C, The cerebella of the DM without (left) and after (right) ablation of the floccular complex (denoted by an asterisk).

ebellar cortex of the DM, because an LM without PNs did not show it. It was previously reported that removal of the disrupted cerebellum of a weaver mutant mouse, in which the majority of cerebellar granule cells are lost, improves motor performance (Grusser and Grusser-Cornehls, 1998). The floccular complex of the cerebellar cortex consisting of flocculus and paraflocculus is implicated in the eye movement controls such as the vestibuloocular reflex and optokinetic response (Robinson, 1981). Therefore, we examined the effect of ablation of this region in DMs. The bilateral flocculectomy (Fig. $3 C$ ) decreased the fast component of spontaneous eye movement (Fig. 3A), abolished the peak around $10 \mathrm{~Hz}$, and decreased the peak around $1 \mathrm{~Hz}$ in the power spectrum (Fig. 3B), suggesting that the floccular complex of a $\mathrm{DM}$ is responsible for the generation of the characteristic fast spontaneous eye movement at $10 \mathrm{~Hz}$.

\section{Firing patterns of action potentials}

The above results suggested that action potential firing patterns of floccular PNs in a DM should have some abnormal components that cause the characteristic spontaneous eye movement, including the $10 \mathrm{~Hz}$ oscillation. Thus, we recorded the spontaneous activities of floccular PNs in alert WMs and DMs (Schiffmann et al., 1999; Goossens et al., 2001). The neuronal activity of a PN was identified by the presence of a characteristic complex spike (Fig. $4 A, D, E$ ) caused by synaptic inputs from a climbing fiber (Ito, 1984), and the recorded site was identified by the fluorescent dye injected from a glass micropipette after recording.

Floccular PNs in WMs showed relatively regular firing of sim- 
A

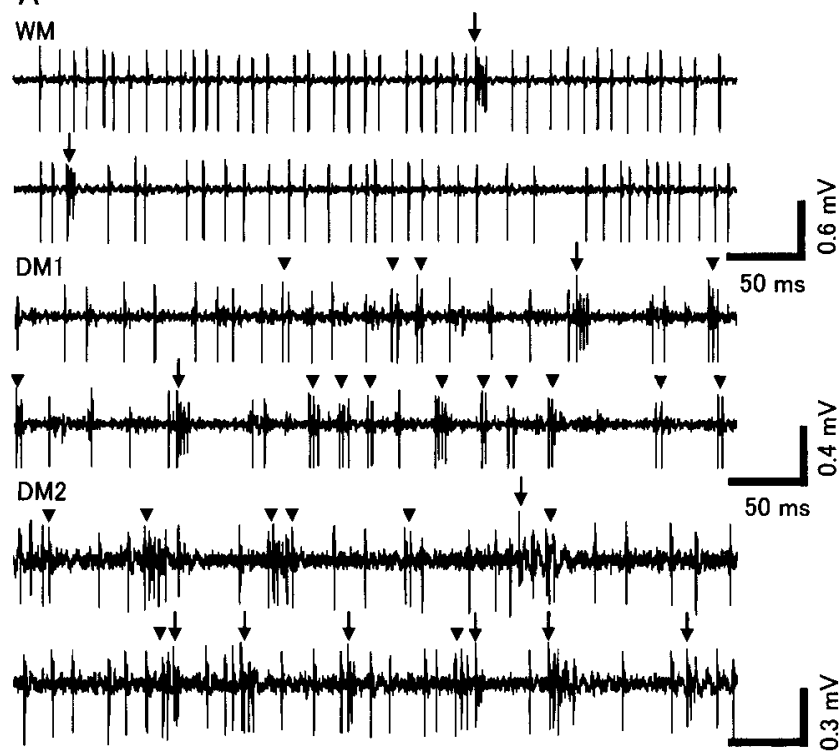

B

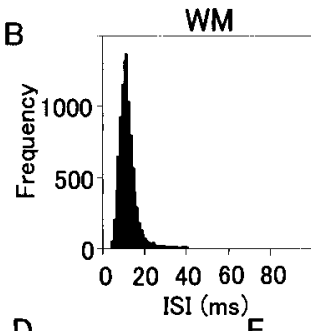

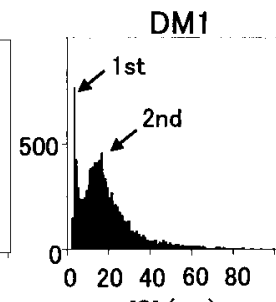

ISI (ms)

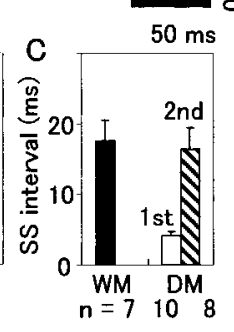

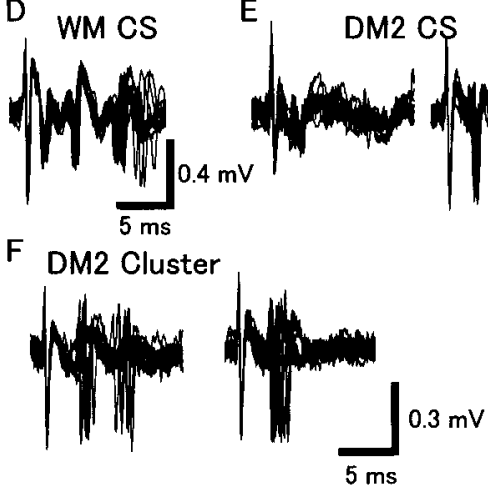

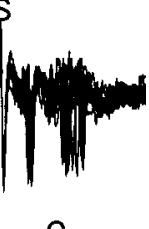

G DM2 SS

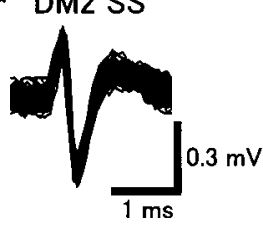

Figure 4. Action potential firing of floccular PNs in a WM and DMs. A, Spontaneous activities of PNs in a WM and DMs (DM1 and DM2). Simple spikes, complex spikes (arrows), and clustered firings (arrowheads) are shown. $B$, Interval histograms of apparent simple spikes in a WM and a DM (DM1). C, The mean \pm SEM of peak intervals of apparent simple spikes is shown. $D, E$, Complex spikes in a WM $(D)$ and a DM ( $E$; DM2). $F, G$, Clustered spikes $(F)$ and simple spikes $(G)$ in the PN of a DM (DM2). Fifteen traces were superimposed in $D-F$, and 100 traces were superimposed in $G$. In the PN of a WM, the time courses of complex spikes were constant. In contrast, multiple time courses of both complex spikes and clustered firings were recorded in the PN of a DM (DM2), although the time courses of simple spikes were constant.

ple spikes (53 $\pm 8 \mathrm{~Hz} ; n=7)$ and occasional complex spikes (mean frequency, $1.1 \pm 0.1 \mathrm{~Hz} ; n=7$ ) (Fig. $4 A$ ). An event consisting of a few spikes with decreasing amplitudes and with intervals of $<5$ msec was identified as a complex spike (Fig. $4 A$, arrows, $D, E$ ). Simple spikes are generated by synaptic inputs from parallel fibers, although it has been reported that an isolated PN fires action potentials regularly (Nam and Hockberger, 1997; Raman and Bean, 1999). The histogram of interspike intervals showed a single peak at $\sim 15 \mathrm{msec}$ in WMs (Fig. $4 B$ ). In contrast, $\mathrm{PNs}$ in DMs sometimes showed repetitive firing of apparent sim-

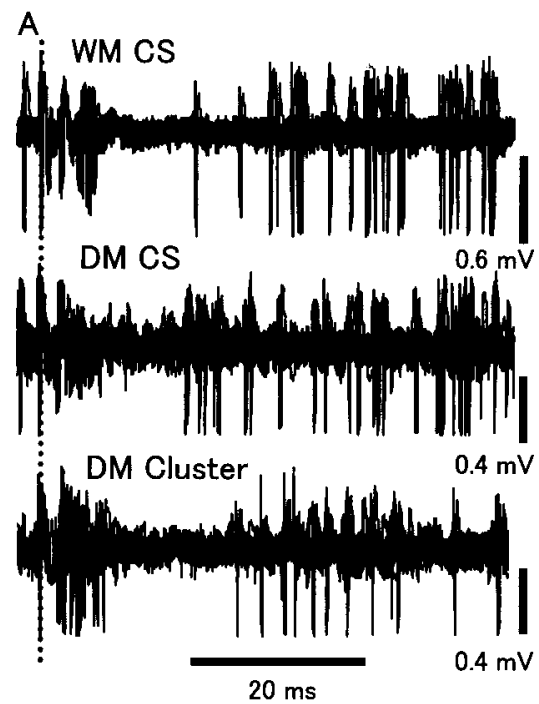

B

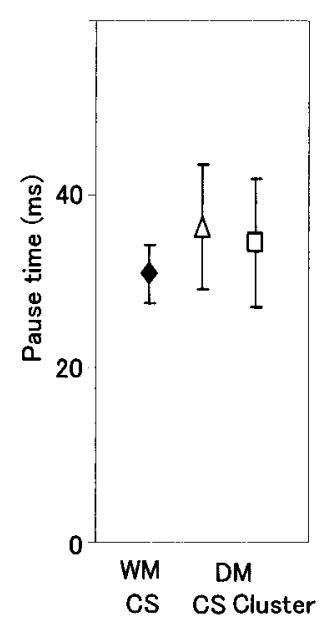

Figure 5. The pose of simple spikes after a complex spike or a clustered firing. A, Fifteen single-unit recording traces after a complex spike or a clustered firing are superimposed for each. The dotted line indicates the onset of a complex spike or a clustered firing. B, The mean \pm SEM of pose times is shown.

ple spikes with short intervals of $<5 \mathrm{msec}$ (Fig. $4 A$, arrowheads, $F)$. Hereafter, such an event is called clustered firing. The tendency that PNs in DMs generate the clustered firing is clearly demonstrated in the interval histogram (Fig. 4B). The distribution of apparent simple spike intervals showed a single peak at $18 \pm 3 \mathrm{msec}$ in WMs $(n=7)$ (Fig. $4 C)$. In contrast, most PNs in DMs (8 of 10) showed two peaks. The shortest peak interval was $4 \pm 1 \mathrm{msec}(n=10)$, and the longer one was $16 \pm 3 \mathrm{msec}(n=8)$ (Fig. 4C). The shortest peak of apparent simple spike intervals in DMs was significantly smaller than that in WMs $(p<0.005$; Student's $t$ test $)$. The average firing frequency $(57 \pm 7 \mathrm{~Hz} ; n=10)$ in DMs was not significantly different from that in WMs. In WMs, the time courses of complex spikes were constant in a PN (Fig. $4 D$ ). In contrast, the time courses of complex spikes were variable in PN of a DM (Fig. 4E). Although they could be classified into several subgroups, in some cases, clear classification was difficult. The quantitative criterion of complex spikes added here was that the peak-to-peak amplitude of the second spike was $<70 \%$ of that of the first one. The mean frequency of complex spikes was $3.3 \pm 0.8 \mathrm{~Hz}(n=10)$ in DMs, which was significantly higher than that in WMs ( $p<0.05$; Student's $t$ test). In a DM, a $\mathrm{PN}$ is innervated by multiple climbing fibers, and some of them innervate distal dendrites (Hashimoto et al., 2001; Ichikawa et al., 2002). Such distal climbing fiber synapses might generate a complex spike that looks different from that in a wild-type PN such as the clustered firing. Actually, the clustered firing sometimes looks like the complex spike (Fig. 4E,F). Presumably, the variation in the time courses of complex spikes was attributable to the multiple innervation of climbing fibers on a PN and to the differences in strength of each climbing fiber input. A relatively weak climbing fiber input might induce variable responses depending on the membrane potential. Although multiple complex spikes can also be recorded when the electrode picks up activities of more than one PN, we think this is unlikely in the analyzed data, because the amplitudes and time courses of simple spikes were constant (Fig. 4G).

It is known that there is a pose period after a complex spike, during which simple spike does not occur (Fig. 5A). This pose time after a complex spike was measured both in DMs and WMs, 

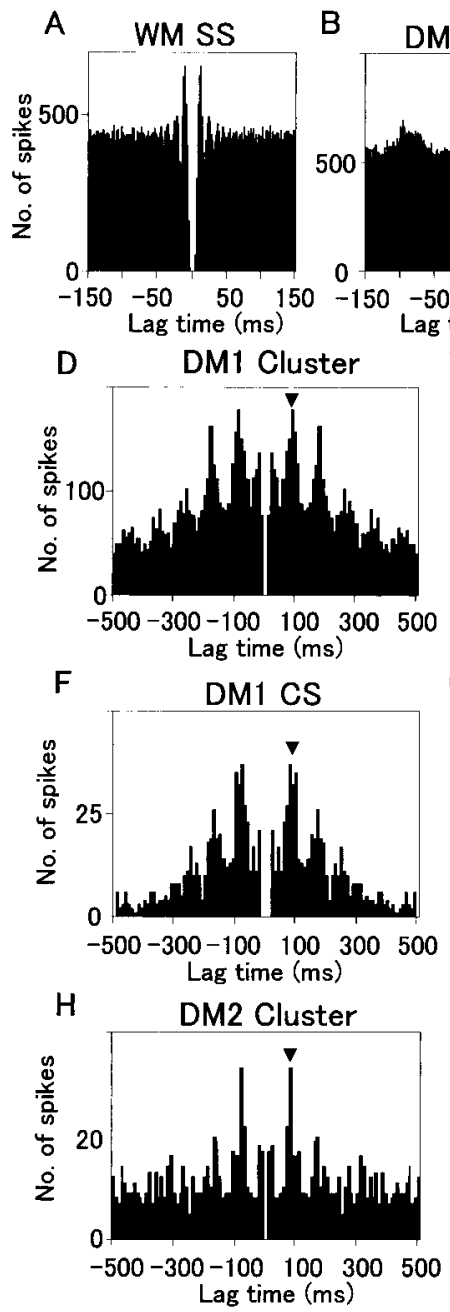

\section{1}

G WM CS
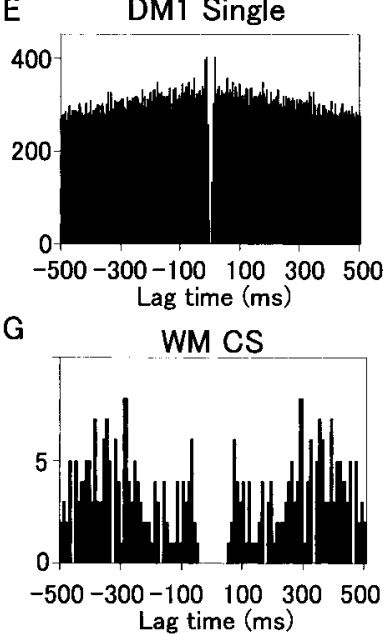

I

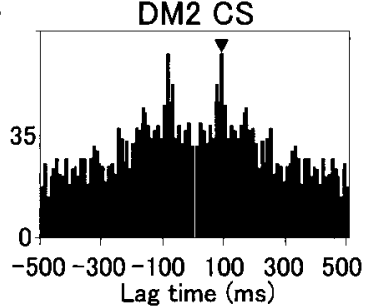

Figure 6. Autocorrelation of simple spikes, complex spikes, and clustered firings. $A, B, A u-$ tocorrelation histograms of apparent simple spikes in PNs of a WM $(A)$ and a DM (DM1) ( $B)$. The arrows indicate the shortest time lag, and the arrowheads indicate the peak $\sim 100 \mathrm{msec}$. Bin width is $1 \mathrm{msec}$. C, The mean \pm SEM of peak lag times is shown. D, The autocorrelation histogram of clustered firings in the PN of a DM. $E$, The autocorrelation histogram of isolated simple spikes that does not constitute clustered firings in a DM. F, G, The autocorrelation histogram of complex spikes in the PNs of a DM $(F)$ and a WM $(G)$. The histograms shown in $B$ and $D-F$ were calculated from the data obtained from the same PN (DM1). H, I, The autocorrelation histograms of clustered firings $(H)$ and complex spikes ( $/$ ) obtained from a PN of another DM (DM2). The bin width is $10 \mathrm{msec}$ in $D-I$. Each histogram was based on the $3 \mathrm{~min}$ recording, except for $G$ (6 min).

and it was also examined whether there was a similar pose period after a clustered firing in DMs. The pose time after a complex spike in WMs and DMs and that after a clustered firing in DMs was $31 \pm 3 \mathrm{msec}(n=7), 36 \pm 7 \mathrm{msec}(n=10)$, and $34 \pm 7 \mathrm{msec}$ $(n=10)$, respectively (Fig. $5 B$ ). Significant differences were not detected among them, suggesting that the clustered firing is also induced by climbing fiber activation. This possibility will be addressed further (see below).

Next, we calculated the autocorrelation of apparent simple spikes. The autocorrelation histograms in WMs showed a clear peak at $18 \pm 3 \mathrm{msec}(n=7)$ and an additional peak at about the double time lag (Fig. 6A). In contrast, those of DMs showed a peak at a very short time lag of $4 \pm 1 \mathrm{msec}(n=10)$ (Fig. $6 B$, arrows) and additional peaks. In some DMs (4 of 10) there was another peak $\sim 100 \mathrm{msec}(85 \pm 4 \mathrm{msec})$ (Fig. $6 B$, arrowheads, $C$ ). The criteria of a peak used here was that the amplitude measured from the mean value of base line was larger than two times the SD of the baseline fluctuations at time lags of 2500-3000 msec (Lang et al., 1997). The existence of a peak $\sim 100 \mathrm{msec}$ in the autocorrelation histogram suggests that the action potential firing of a PN has a periodicity of $\sim 10 \mathrm{~Hz}$ in DMs, which corresponds to the frequency of fast spontaneous eye movement. The $10 \mathrm{~Hz}$ oscillatory activity and the tendency to generate clustered firing were also observed in PNs in other regions of the cerebellar cortex of DMs. The peak $\sim 100$ msec has never been detected in WMs ( 0 of 7).

Both the clustered firing and the $10 \mathrm{~Hz}$ oscillation were recorded only in DMs, which suggested that these two phenomena might be interrelated. Thus, we calculated the autocorrelation of clustered firings in DMs. The autocorrelation histograms showed peaks at $\sim 100 \mathrm{msec}(88 \pm 4 \mathrm{msec})$ and its multiples ( 6 of 10 cases) (Fig. $6 D, H$ ). We also calculated the autocorrelation of simple spikes that do not constitute clustered firings. There was no clear $100 \mathrm{msec}$ peak in the histogram (Fig. 6E). These results suggest that the $10 \mathrm{~Hz}$ oscillation in the action potential firing of the PN of a DM is attributable to the clustered firings. The autocorrelation histogram of clustered firings also tended to show a peak $\sim 1$ sec in some DMs (5 of 10 cases; data not shown). Because clustered firings were likely to be induced by climbing fibers, we next compared the autocorrelation histograms of clustered firings with those of complex spikes in DMs. The latter also showed a similar peak at $\sim 100 \mathrm{msec}(81 \pm 3 \mathrm{msec}$ ) and its multiples ( 7 of 10 cases) (Fig. $6 F, I$ ) and the tendency to make another peak $\sim 1$ $\sec (7$ of 10 cases; data not shown), suggesting a common mechanism to generate clustered firings and complex spikes. In WMs, the autocorrelation histogram of complex spikes did not show clear peaks (Fig. 6G).

Complex spikes and clustered firings in the cerebellar slices To further examine the idea that the clustered firing is induced by climbing fiber activation, we applied the patch-clamp technique to cerebellar slices prepared from WMs and DMs. First, spontaneous action potential firing was recorded extracellularly from a $\mathrm{PN}$ in a cell-attached mode at room temperature. Although PNs showed spontaneous action potential firing, they showed neither the clustered firing nor oscillatory activity both in WMs and DMs ( $n=17$ for each). The interval histograms of action potentials showed only one peak in both WMs and DMs (Fig. 7A), and the autocorrelation histograms also showed peaks corresponding to the peak of the interval histogram and its multiples (data not shown). The spike intervals tended to be longer and more variable in DMs. The mean spike intervals were $101 \pm 73 \mathrm{msec}$ (mean $\pm \mathrm{SD} ; n=17)$ in WMs and $166 \pm 210 \mathrm{msec}(n=17)$ in DMs (Fig. 7B). We did not record spontaneous complex spikes in slice preparations prepared from both WMs and DMs. These results suggest that intact neuronal circuits are required for the clustered firing, oscillation, and spontaneous synaptic inputs from climbing fibers in DMs.

We next recorded climbing fiber responses in PNs in a wholecell patch-clamp condition. First, a climbing fiber-induced EPSC was identified by the paired-pulse depression in a voltage-clamp condition (Fig. 7C). Previous studies showed that when input fibers are stimulated twice with a certain interval, the second EPSC is smaller than the first at the climbing fiber-PN synapses (paired-pulse depression), whereas the second is larger than the first at the parallel fiber-PN synapses (paired-pulse potentiation) (Konnerth et al., 1990; Kashiwabuchi et al., 1995). After confirming that a climbing fiber was stimulated with the paired-pulse depression, the recording condition was changed from a voltageclamp to a current-clamp condition, and the voltage response to activation of a climbing fiber was recorded (Fig. 7D). In a WM, a 

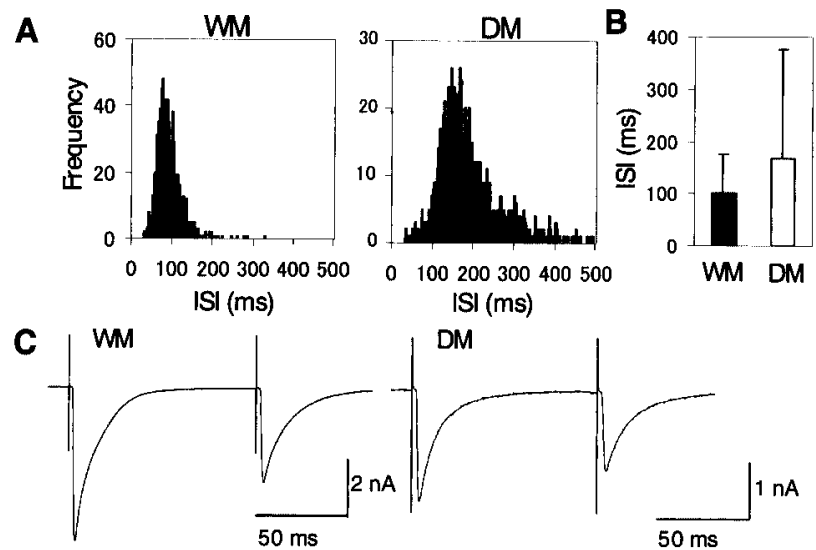

$\mathrm{ISI}(\mathrm{ms})$
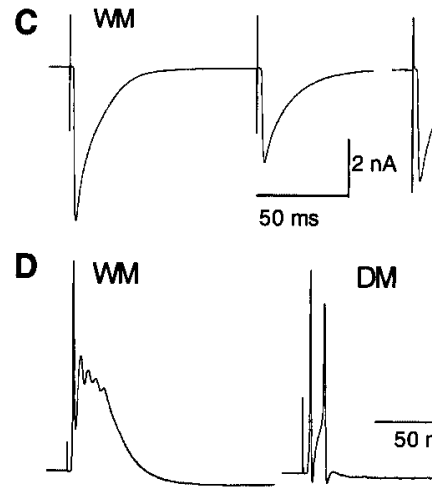

$\mathrm{DM}$
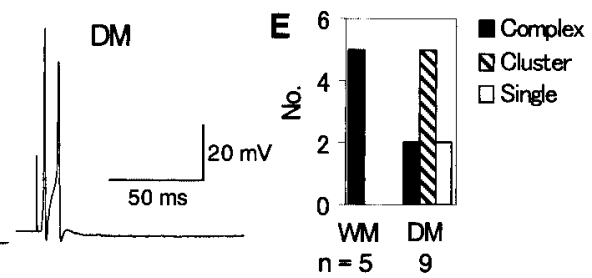

Figure 7. Patch-clamp recording in slice preparations. $A$, Interval histograms of action potentials recorded extracellularly from PNs in slices prepared from a WM and a DM. $B$, The mean $\pm S D$ of peak intervals is shown. The larger variation in the interval histograms was noticed in DMs. C, EPSCs induced by activation of a climbing fiber were identified by the pairedpulse depression. $D$, Climbing fiber responses in a current-clamp condition in a WM and a DM.E, The numbers of complex spikes, clustered firings, and single spikes induced by climbing fiber activation are shown.

typical complex spike with a full-sized action potential, followed by smaller depolarization (Llinas and Sugimori, 1980), was recorded (5 of 5). In contrast, almost full-sized action potentials with short intervals (clustered firing) were induced by climbing fiber activation in some PNs in DMs (Fig. 7D). Here, a series of action potentials with short intervals and with the amplitude of the second spike $>70 \%$ of that of the first spike was identified as a clustered firing. In DMs, climbing fiber activation induced a clustered firing in five of nine cases, a complex spike in two of nine cases, and a single spike in two of nine cases (Fig. $7 E$ ). Thus, the clustered firing was evoked by climbing activation in DMs, suggesting that at least some of the clustered firings recorded in vivo DMs were, indeed, unordinary complex spikes.

\section{Simultaneous recording of the single-unit activity and the eye position}

The above results suggest that the oscillatory climbing fiber activity inducing complex spikes and clustered firings in PNs is a cause of spontaneous $10 \mathrm{~Hz}$ eye movement in a DM. To examine the correlation between the climbing fiber activity and the eye movement directly, we performed simultaneous recordings of the single PN activity and the eye position in DMs. Although the slow sampling frequency of the eye position recording system hampered the precise timing adjustment, the correlation was observed between the spontaneous eye movement and complex spikes and clustered firings ( 6 of 8 and 5 of 8 cases, respectively) (Fig. 8). Both complex spikes and clustered firings tended to fire during a particular phase of slow oscillatory eye movement $\sim 1$ Hz. They preferentially fired when the eye was in the ipsilateral position in the horizontal movement or in a lower position in the vertical movement. The averaged eye movement traces triggered by either a complex spike or a clustered firing showed the slow
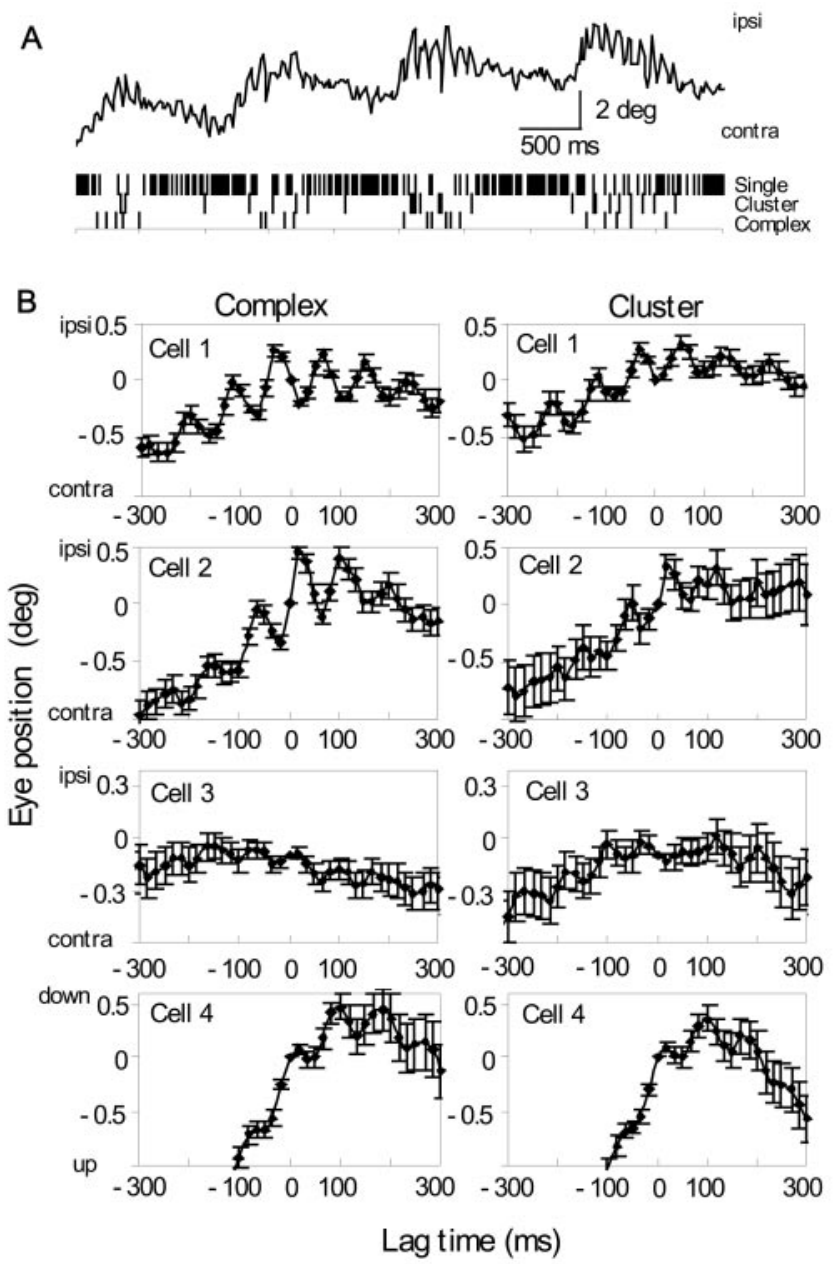

Figure 8. Simultaneous recording of the eye movement and the single PN activity. $A$, The top trace shows the eye position. The bottom vertical bars indicate the timings of complex spikes, clustered firings, and isolated simple spikes that do not constitute clustered firings. $B$, The averaged traces of eye position triggered by a complex spike or by a clustered firing occurring at time 0 . Cells $1-3$ were correlated to the horizontal eye movement, and cell 4 to the vertical movement. Cell 3 did not show a clear correlation. The eye position at 0 msec in each trace was set at $0^{\circ}$ before averaging. Seventy to 120 traces were averaged. The SEM is also shown.

$(\sim 1 \mathrm{~Hz})$ and fast $(\sim 10 \mathrm{~Hz})$ oscillation (Fig. $8 B)$. These results also suggest that the rhythmic climbing fiber activity is related to the oscillatory spontaneous eye movement in a DM. The extent of correlation between the eye movement and complex spike or clustered firing was variable. Some showed relatively clear correlation (Fig. 8B, cells 1, 2 , and 4), and the other (cell 3) did little. These results might suggest that multiple climbing fibers carrying various information form synapses on floccular PNs in DMs. The $10 \mathrm{~Hz}$ oscillatory eye movement might be induced by ensemble activities of the population of inferior olivary neurons as in the licking behavior of rats (Welsh et al., 1995).

\section{Discussion}

Our results indicate that certain motor control ability is retarded more severely in a DM than in an LM despite relatively normal cerebellar morphology and the presence of PNs in a DM. Lesioning the floccular complex of the cerebellum in a DM removed the fast $10 \mathrm{~Hz}$ component of surplus eye movement and decreased the slow component, suggesting that a part of the cerebellar cortex in a DM outputs inappropriate neuronal signals responsible for the abnormal motion. Both complex spikes and clustered 
firings were shown to oscillate at $\sim 10 \mathrm{~Hz}$ in a floccular $\mathrm{PN}$ in a DM. It was suggested that not only a complex spike but also a clustered firing is induced by the climbing fiber activation in a DM. Finally, simultaneous recording of the single PN activity and the eye movement in a DM showed that complex spikes and clustered firings were correlated to the spontaneous eye movement. These results suggest that the failures in synaptic regulation because of the GluR $\delta 2$ deficiency produce abnormal oscillating neuronal signals, depending on the climbing fiber activity, that result in the generation of surplus involuntary movement and influence motor performance even worse than no signal at all.

The notion that aberrant cerebellar output is functionally worse than no cerebellar output has been proposed. In a Weaver mutant mouse, in which massive degeneration of cerebellar granule cells occurs together with degeneration of dopaminergic cells in substantia nigra, the lesion of cerebellum improved motor performance (Grusser and Grusser-Cornehls, 1998; GrusserCornehls and Baurle, 2001). Similar improvement of motor performance after the cerebellectomy was also reported in genetically dystonic rats (LeDoux et al., 1993). These results suggest that faulty cerebellar outputs are better to be removed. Our results are in accordance with these studies and have extended in the following respects. First, in a DM and an LM, the mutated gene is known $(G l u R \delta 2)$, and it is selectively expressed in PNs. Thus, in these mutant mice, the origin of abnormality is restricted to cerebellar PNs. Second, we compared the motor performance of intact DMs not only with that of cerebellectomized DMs but also with that of LMs, in which all PNs degenerate because of the mutation in the same GluR $\delta 2$ gene. Our study also restricted the lesioned area to small parts of the cerebellar cortex. Third, we have analyzed motor performance quantitatively and showed the $10 \mathrm{~Hz}$ oscillation in the spontaneous eye movement. Fourth, we have demonstrated the rhythmic PN activities correlated with the abnormal motor oscillation and analyzed the cause of such activity.

The flocculectomy and in vivo recording of floccular PN activity suggested that floccular outputs are responsible for the 10 and $1 \mathrm{~Hz}$ spontaneous eye movement. Then, how do floccular PNs induce the eye movement? Floccular PNs are targeting neurons in the vestibular nucleus that directly regulate activity of motor neurons of extra-ocular muscles (Robinson, 1981). GABAergic inhibitory activity of PNs should inhibit spontaneous activity of the vestibular nucleus neurons, which suppress activity of the motor neurons, resulting in the eye ball movement through suppression of tonic contraction of the muscle.

The mechanism underlying the abnormal spike firing pattern of PNs in a DM has been addressed here. The clustered firings and complex spikes were shown to oscillate at $\sim 10 \mathrm{~Hz}$. We have suggested that both complex spikes and clustered firings are generated by climbing fiber activity. The reasons are: (1) a similar pose time of simple spikes $(\sim 30 \mathrm{msec})$ followed both complex spikes and clustered firings; (2) both complex spikes and clustered firings showed similar autocorrelation histograms with the $10 \mathrm{~Hz}$ oscillation; (3) climbing fiber activation induced both of them in slice preparations; (4) both are correlated to the spontaneous eye movement to a similar extent; and (5) in a DM, multiple climbing fibers are innervating to a $\mathrm{PN}$ and some form synapses on distal dendrites (Kurihara et al., 1997; Hashimoto et al., 2001; Ichikawa et al., 2002). In DMs, the extent of correlation of clustered firings or complex spikes with the eye movement was variable. We presume that such variability is attributable to the variation of properties of climbing fibers innervating to PNs, although the possibility that some factors other than those di- rectly related to climbing fiber activity contribute to the generation of clustered firings cannot be excluded. In any case, the activity of inferior olivary neurons seems to be responsible for the $10 \mathrm{~Hz}$ oscillation at least partly.

Previous studies demonstrated that inferior olivary neurons show intrinsic membrane oscillation at several Hertz (Llinas and Yarom, 1986) and that the spontaneous complex spike activity in an alert rat shows the $10 \mathrm{~Hz}$ rhythmicity (Lang et al., 1999). Approximate $10 \mathrm{~Hz}$ periodicity of the animal movement has been known, and the importance of physiological tremor has been recognized for a long time. It is also reported that activation of inferior olivary nuclei by systemic application of harmaline induces rhythmic activation of muscles throughout the body, which is observed as a $10 \mathrm{~Hz}$ tremor (Welsh and Llinas, 1997). Rhythmic complex spike activity has been related to the pulsate organization of licking movement in rats (Welsh et al., 1995). Taking all these previous results into account, it seems that enhanced rhythmic activity of inferior olivary neurons is responsible for the $\sim 10 \mathrm{~Hz}$ oscillation in the spontaneous eye movement in a DM. Presumably, in a DM the intrinsic rhythmic property of an inferior olivary neuron becomes more influential to the PN activity.

Then, what makes climbing fiber activity more influential on the PN activity in a DM? In a DM, a PN is innervated by multiple climbing fibers (Kurihara et al., 1997; Hashimoto et al., 2001; Ichikawa et al., 2002) that are likely to increase the number of synaptic inputs from climbing fibers and, thus, the $\mathrm{PN}$ responses to them. It is also known that the number of parallel fiber-PN synapses is reduced in a DM, which should decrease the contribution of parallel fiber inputs on the PN activity, thus increasing the relative contribution of climbing fiber inputs. Finally, the lack of LTD in a DM might also contribute. The LTD has been considered to contribute to motor learning. Actually, a DM shows deficits in both motor learning and motor coordination (Kashiwabuchi et al., 1995; Funabiki et al., 1995; Kishimoto et al., 2001), which should increase error signals in the motor control system. It has been proposed that inferior olivary neurons code error signals; in particular, climbing fiber inputs to the cerebellar flocculus are reported to code retinal slip errors (Ito, 2001). The result that the $10 \mathrm{~Hz}$ oscillatory eye movement in a DM was less clear in the dark might also support this idea, because there should be no visual error signal in the dark. Thus, the motor discoordination and motor learning deficits might increase the climbing fiber activity in a DM.

Taken together, we suggest the following scenario for the severe motor discoordination in a DM (Fig. 9). The lack of GluR $\delta 2$ causes multiple climbing fiber innervation and a reduction in the number of parallel fiber-PN synapses, both of which work to increase the relative contribution of climbing fiber activity on a $\mathrm{PN}$. The LTD failure in a DM might also increase the climbing fiber activity coding the error signal through the motor learning deficit. All these contribute to increase the influence of climbing fiber activity with the intrinsic $10 \mathrm{~Hz}$ rhythm on a PN, which in turn causes the involuntary surplus movement and disturbs the motor control.

However, our results also suggest that the above scheme might not be the whole story. Spontaneous eye movement remained in the dark or after ablation of the flocculus, although the $10 \mathrm{~Hz}$ oscillation disappeared. These results might suggest that the enhancement of rhythmic climbing fiber inputs to PNs by itself is not the sole cause of the involuntary movement in a DM. Other plastic changes might be induced in both the cerebellum and the brainstem. Several types of synaptic plasticity other than LTD 


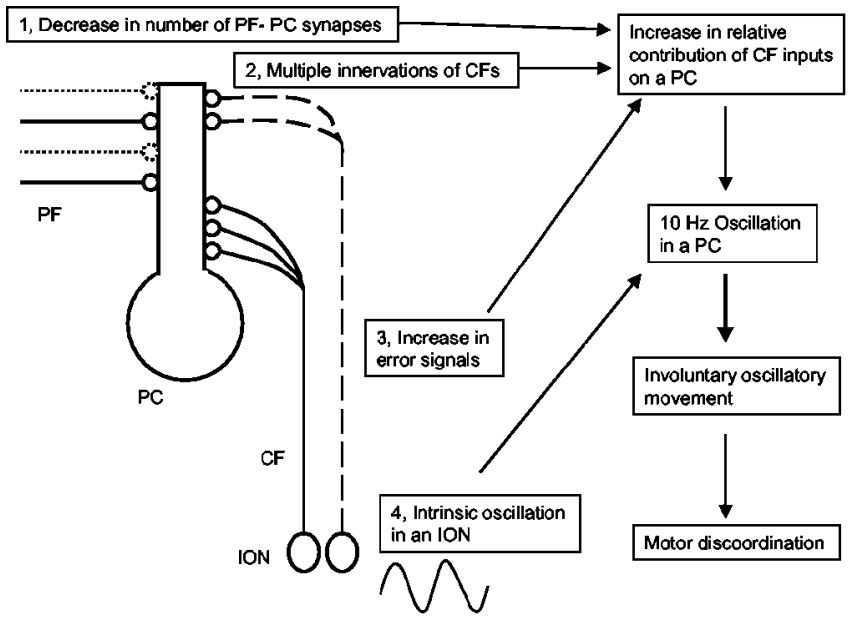

Figure 9. A scheme explaining the cause of $10 \mathrm{~Hz}$ oscillation in the PN activity and the motor discoordination. PF, Parallel fiber; CF, climbing fiber; ION, inferior olivary neuron.

have been reported in both the cerebellar cortex and nuclei (Kano et al., 1992; Morishita and Sastry, 1996; D’Angelo et al., 1999; Hansel and Linden, 2000; Kawaguchi and Hirano, 2000; Hansel et al., 2001), and they might be induced or influenced by the altered PN activity in a DM. Alternatively, intrinsic excitability of neurons in the cerebellum, brainstem, or both might also be changed (Desai et al., 1999; Aizenman and Linden, 2000). In any case, the GluR $\delta 2$ deficiency seems to cause substantial changes in the function of cerebellar cortex and related structures.

\section{References}

Aiba A, Kano M, Chen C, Stanton ME, Fox GD, Herrup K, Zwingman TA, Tonegawa S (1994) Deficient cerebellar long-term depression and impaired motor learning in mGluR1 mutant mice. Cell 7:377-388.

Aizenman CD, Linden DJ (2000) Rapid, synaptically driven increases in the intrinsic excitability of cerebellar deep nuclear neurons. Nat Neurosci 3:109-111.

Araki K, Meguro H, Kushiya E, Takayama C, Inoue Y, Mishina M (1993) Selective expression of the glutamate receptor channel $\delta 2$ subunit in cerebellar Purkinje cells. Biochem Biophys Res Commun 197:1267-1276.

Chen C, Kano M, Abeliovich A, Chen L, Bao S, Kim JJ, Hashimoto K, Thompson RF, Tonegawa S (1995) Impaired motor coordination correlates with persistent multiple climbing fiber innervation in PKC $\gamma$ mutant mice. Cell 83:1233-1242.

Conquet F, Bashir ZI, Davies CH, Daniel H, Ferraguti F, Bordi F, Franz-Bacon K, Reggiani A, Matarese V, Conde F, Collingridge GL, Crepel F (1994) Motor deficit and impairment of synaptic plasticity in mice lacking mGluR1. Nature 372:237-243.

D’Angelo E, Rossi P, Armano S, Taglietti V (1999) Evidence for NMDA and mGluR receptor-dependent long-term potentiation of mossy fibregranule cell transmission in rat cerebellum. J Neurophysiol 81:277-287.

Desai NS, Rutherford LC, Turrigiano GG (1999) Plasticity in the intrinsic excitability of cortical pyramidal neurons. Nat Neurosci 2:515-520.

De Zeeuw CI, Hansel C, Bian F, Koekkoek SK, van Alphen AM, Linden DJ, Oberdick J (1998) Expression of a protein kinase C inhibitor in Purkinje cells blocks cerebellar LTD and adaptation of the vestibulo-ocular reflex. Neuron 20:495-508.

Fortier PA, Smith AM, Rossignol S (1987) Locomotor deficits in the mutant mouse, Lurcher. Exp Brain Res 66:271-286.

Funabiki K, Mishina M, Hirano T (1995) Retarded vestibular compensation in mutant mice deficient in $\delta 2$ glutamate receptor subunit. NeuroReport 7:189-192.

Goossens J, Daniel H, Rancillac A, van der Steen J, Oberdick J, Crepel F, De Zeeuw CI, Frens MA (2001) Expression of protein kinase C inhibitor blocks cerebellar long-term depression without affecting Purkinje cell excitability in alert mice. J Neurosci 21:5813-5823.

Grusser C, Grusser-Cornehls U (1998) Improvement in motor perfor- mance of Weaver mutant mice following lesions of the cerebellum. Behav Brain Res 97:189-194.

Grusser-Cornehls U, Baurle J (2001) Mutant mice as a model for cerebellar ataxia. Prog Neurobiol 63:489-540.

Hansel C, Linden DJ (2000) Long-term depression of the cerebellar climbing fiber-Purkinje neuron synapse. Neuron 26:473-482.

Hansel C, Linden DJ, D’Angelo E (2001) Beyond parallel fiber LTD: the diversity of synaptic and non-synaptic plasticity in the cerebellum. Nat Neurosci 4:467-475.

Hashimoto K, Ichikawa R, Takechi H, Inoue Y, Aiba A, Sakimura K, Mishina M, Hashikawa T, Konnerth A, Watanabe M, Kano M (2001) Roles of glutamate receptor $\delta 2$ subunit (GluR $\delta 2)$ and metabotropic glutamate receptor subtype 1 (mGluR1) in climbing fiber synapse elimination during postnatal cerebellar development. J Neurosci 21:9701-9712.

Hilber P, Caston J (2001) Motor skills and motor learning in Lurcher mutant mice during aging. Neuroscience 102:615-623.

Hirano T, Kasono K, Araki K, Shinozuka K, Mishina M (1994) Involvement of the glutamate receptor $\delta 2$ subunit in the long-term depression of glutamate responsiveness in cultured rat Purkinje cells. Neurosci Lett 182:172-176.

Hirano T, Kasono K, Araki K, Mishina M (1995) Suppression of LTD in cultured Purkinje cells deficient in the glutamate receptor $\delta 2$ subunit. NeuroReport 6:524-526.

Hollmann M, Heinemann S (1994) Cloned glutamate receptors. Annu Rev Neurosci 17:31-108.

Ichikawa R, Miyazaki T, Kano M, Hashikawa T, Tatsumi H, Sakimura K, Mishina M, Inoue Y, Watanabe M (2002) Distal extension of climbing fiber territory and multiple innervation caused by aberrant wiring to adjacent spiny branchlets in cerebellar Purkinje cells lacking glutamate receptor $\delta 2$. J Neurosci 22:8487-8503.

Ito M (1984) The cerebellum and neural control. New York: Raven.

Ito M (2001) Cerebellar long-term depression: characterization, signal transduction, and functional roles. Physiol Rev 81:1143-1195.

Iwashita M, Kanai R, Funabiki K, Matsuda K, Hirano T (2001) Dynamic properties, interactions and adaptive modifications of vestibulo-ocular reflex and optokinetic response in mice. Neurosci Res 3:299-311.

Jeromin A, Huganir RL, Linden DJ (1996) Suppression of the glutamate receptor $\delta 2$ subunit produces a specific impairment in cerebellar longterm depression. J Neurophysiol 76:3578-3583.

Kano M, Rexhausen U, Dreessen J, Konnerth A (1992) Synaptic excitation produces a long- lasting rebound potentiation of inhibitory synaptic signals in cerebellar Purkinje cells. Nature 356:601-604.

Kashiwabuchi N, Ikeda K, Araki K, Hirano T, Shibuki K, Takayama C, Inoue Y, Kutsuwada T, Yagi T, Kang Y, Aizawa S, Mishina M (1995) Impairment of motor coordination, Purkinje cell synapse formation, and cerebellar long-term depression in GluR $\delta 2$ mutant mice. Cell 81:245-252.

Katoh A, Kitazawa H, Itohara S, Nagao S (2000) Inhibition of nitric oxide synthesis and gene knockout of neuronal nitric oxide synthase impaired adaptation of mouse optokinetic response eye movements. Learn Mem $7: 220-226$.

Kawaguchi S, Hirano T (2000) Suppression of inhibitory synaptic potentiation by presynaptic activity through postsynaptic $\mathrm{GABA}_{\mathrm{B}}$ receptors in a Purkinje neuron. Neuron 27:339-347.

Kishimoto Y, Kawahara S, Suzuki M, Mori H, Mishina M, Kirino Y (2001) Classical eyeblink conditioning in glutamate receptor subunit $\delta 2$ mutant mice is impaired in the delay paradigm but not in the trace paradigm. Eur J Neurosci 13:1249-1253.

Kohda K, Wang Y, Yuzaki M (2000) Mutation of a glutamate receptor motif reveals its role in gating and $\delta 2$ receptor channel properties. Nat Neurosci 3:315-322.

Konnerth A, Llano I, Armstrong CM (1990) Synaptic currents in cerebellar Purkinje cells. Proc Natl Acad Sci USA 87:2662-2665.

Kurihara H, Hashimoto K, Kano M, Takayama C, Sakimura K, Mishina M, Inoue Y, Watanabe M (1997) Impaired parallel fiber $\rightarrow$ Purkinje cell synapse stabilization during cerebellar development of mutant mice lacking the glutamate receptor $\delta 2$ subunit. J Neurosci 17:9613-9623.

Lang EJ, Sugihara I, Llinas R (1997) Differential roles of apamin- and charybdotoxin-sensitive $\mathrm{K}^{+}$conductances in the generation of inferior olive rhythmicity in vivo. J Neurosci 15:2825-2838.

Lang EJ, Sugihara I, Welsh JP, Llinas R (1999) Patterns of spontaneous Purkinje cell complex spike activity in the awake rat. J Neurosci 19:2728-2739. 
LeDoux MS, Lorden JF, Ervin JM (1993) Cerebellectomy eliminates the motor syndrome of the genetically dystonic rat. Exp Neurol 120:302-310.

Linden DJ, Connor JA (1995) Long-term synaptic depression. Annu Rev Neurosci 18:319-359.

Llinas R, Sugimori M (1980) Electrophysiological properties of in vitro Purkinje cell somata in mammalian cerebellar slices. J Physiol (Lond) 305:171-195.

Llinas R, Yarom Y (1986) Oscillatory properties of guinea-pig inferior olivary neurons and their pharmacological modulation: an in vitro study. J Physiol (Lond) 376:163-182.

Lomeli H, Sprengel R, Laurie DJ, Kohr G, Herb A, Seeburg PH, Wisden W (1993) The rat $\delta$ - 1 and $\delta$-2 subunits extend the excitatory amino acid receptor family. FEBS Lett 315:318-322.

Morishita W, Sastry BR (1996) Postsynaptic mechanism underlying longterm depression of GABAergic transmission in neurons of the deep cerebellar nuclei. J Neurophysiol 76:59-68.

Nam SC, Hockberger PE (1997) Analysis of spontaneous electrical activity in cerebellar Purkinje cells acutely isolated from postnatal rats. J Neurobiol 33:18-32.

Raman IM, Bean BP (1999) Ionic currents underlying spontaneous action potentials in isolated cerebellar Purkinje neurons. J Neurosci 19:1663-1674.

Raymond JL, Lisberger SG, Mauk MD (1996) The cerebellum: a neuronal learning machine? Science 272:1126-1131.

Robinson DA (1981) Control of eye movement. In: Handbook of physiology, Sect 1 (Brooks V, ed), pp 1275-1320. Bethesda, MD: Williams and Wilkins.

Rosenmund C, Stern-Bach Y, Stevens CF (1998) The tetrameric structure of a glutamate receptor channel. Science 280:1596-1599.
Schiffmann SN, Cheron G, Lohof A, d'Alcantara P, Meyer M, Parmentier M, Schurmans S (1999) Impaired motor coordination and Purkinje cell excitability in mice lacking calretinin. Proc Natl Acad Sci USA 96:5257-5262.

Shibuki K, Gomi H, Chen L, Bao S, Kim JJ, Wakatsuki H, Fujisaki T, Fujimoto K, Katoh A, Ikeda T, Chen C, Thompson RF, Itohara S (1996) Deficient cerebellar long-term depression, impaired eyeblink conditioning, and normal motor coordination in GFAP mutant mice. Neuron 16:587-599.

Stahl JS, van Alphen AM, De Zeeuw CI (2000) A comparison of video and magnetic search coil recordings of mouse eye movements. J Neurosci Methods 99:101-110.

Swisher DA, Wilson DB (1977) Cerebellar histogenesis in the lurcher (Lc) mutant mouse. J Comp Neurol 17:205-218.

Takayama C, Nakagawa S, Watanabe M, Mishina M, Inoue Y (1996) Developmental changes in expression and distribution of the glutamate receptor channel $\delta 2$ subunit according to the Purkinje cell maturation. Brain Res Dev Brain Res 92:147-155.

Welsh JP, Llinas R (1997) Some organization principles for the control of movement based on olivocerebellar physiology. Prog Brain Res 114:449-461.

Welsh JP, Lang EJ, Sugihara I, Llinas R (1995) Dynamic organization of motor control within the olivocerebellar system. Nature 374:453-457.

Zhao HM, Wenthold RJ, Wang YX, Petralia RS (1997) $\delta$-glutamate receptors are differentially distributed at parallel and climbing fiber synapses on Purkinje cells. J Neurochem 68:1041-1052.

Zuo J, De Jager PL, Takahashi KA, Jiang W, Linden DJ, Heintz N (1997) Neurodegeneration in Lurcher mice caused by mutation in $\delta 2$ glutamate receptor gene. Nature 388:769-773. 